\title{
Análisis de la justicia restaurativa para atender casos de violencia intrafamiliar en el Centro de Atención Integral a Víctimas de Violencia Intrafamiliar (CAVIF) de la Fiscalía General de la Nación, Colombia*
}

\section{Analysis of restorative justice attending cases of Intrafamiliar Violence in the Integral Centre for Victims of Domestic Violence (CAVIF) - Fiscalía General de la Nación, Colombia}

\author{
Leonardo Alberto Rodríguez Cely** \\ Andrea Padilla Villarraga \\ Luz Stella Rodríguez \\ Fernando Díaz Colorado \\ Pontificia Universidad Javeriana, \\ Colombia
}

Recibido: 25 de enero de 2010

Revisado: 20 de febrero de 2010

Aceptado: 15 de abril de 2010

\section{Resumen}

La presente investigación hace un análisis de la Justicia Restaurativa orientado a la atención de casos de violencia intrafamiliar en el Centro de Atención Integral a Víctimas de Violencia Intrafamiliar (CAVIF) de la Fiscalía General de la Nación. Este análisis es el producto de un trabajo conceptual y un ejercicio metodológico aplicado a un proceso de recolección, sistematización y análisis de la información, basado en un diseño de investigación exploratoria. Para el estudio se tuvo una muestra intencional, integrada por 20 usuarias y 18 profesionales del CAVIF, a quienes se les administraron entrevistas a profundidad, diseñadas a partir de categorías previamente definidas. De esta manera, se describe una serie de necesidades del contexto institucional, profesional y de los propios usuarios y funcionarios del sistema, las cuales permiten comprender las condiciones necesarias, para proponer unos lineamientos generales para la formulación de un programa de justicia restaurativa, acorde con la ley vigente en Colombia.

Artículo de investigación. Grupo de Investigación. Psicología Jurídica y Forense. Retos y Perspectivas. El estudio es subvencionado por la Vicerrectoría Académica de la Pontificia Universidad Javeriana.

Correspondencia: Leonardo Alberto Rodríguez Cely, Facultad de Psicología, Pontificia Universidad Javeriana, Colombia. Cra. 5 No. 39 - 00, piso 2, Bogotá, Colombia. Correo electrónico: lacely@javeriana.edu.co. 
Los resultados del estudio permiten señalar la necesidad de llevar a cabo procesos de capacitación en justicia restaurativa con los funcionarios responsables de dar trámite a los conflictos de violencia intrafamiliar en el CAVIF. Como aporte final se proponen criterios para ejecutar un programa de justicia restaurativa.

Palabras clave: justicia restaurativa, victimología, violencia intrafamiliar.

\section{Abstract}

The actual investigation makes an emphasis about restorative justice, its oriented to catch the attention for those cases of inner familiar violence, cases that are being taking care of the integral centre attention for victims of domestic violence (CAVIF). Existence of the following analysis appears on the basis of conceptual work and methodological exercise, all applied from a process of collecting, systematizing and analyzing information based on a design of exploratory investigation. Study had an intentional sample, 20 female users and 18 professionals from (CAVIF) proceeded with a series of profound interviews designed based on categories previously defined. This way it's described all kind of context needs, institutional, professional and own users and functionaries of the system in order to comprehend the necessary conditions, proposing general alignments for the formulation of a restorative justice on accordance of actual by law in Colombia. Results of the study are permitting point to the necessity of carry on different process of capacitating the restorative justice functionaries, responsible for allowing the necessary steps to the domestic violence conflicts in the (CAVIF). As a last it is a criteria proposed in order to carry on a justice restorative program.

Key words: Justice Restorative, Victimology, and Violence Domestic.

El replanteamiento de las prácticas de administración de justicia a nivel internacional, el desarrollo de un sistema penal acusatorio en Colombia (Ley 906 de 2004), el Código de Infancia y Adolescencia (Ley 1098 de 2006) y las nuevas disposiciones en derecho de familia han tenido efectos como la ampliación de la institucionalidad y una mayor oferta pública alternativa y complementaria al sistema tradicional de justicia. La ampliación de la oferta de mecanismos alternativos al sistema penal retributivo, impulsada como parte de una política nacional orientada al resarcimiento de los derechos de las víctimas, al empoderamiento de la comunidad y a la generación de alternativas a la judicialización de los conflictos, ha estado acompañada de una institucionalidad integrada de apoyo, representada en la creación de nuevas figuras y servicios que deben estar acordes con los planteamientos y requerimientos del nuevo paradigma restaurativo. Al igual que otros con- flictos, la violencia intrafamiliar ha comenzado a hacer parte de esta nueva concepción de justicia, por lo cual las instituciones correspondientes han entrado a diseñar e implementar programas alternativos.

En este sentido, la Fiscalía General de la Nación ha creado el Centro de Atención Integral a Víctimas de Violencia Intrafamiliar (CAVIF), con el fin de "restaurar familias en situación de violencia intrafamiliar y sexual, generando una sinergia entre la infraestructura de justicia ya creada y [el] CAVIF" (Fiscalía General de la Nación, Acuerdo 123 de 2004). De acuerdo con el documento base de la entidad, el servicio brindado en la institución es "un modelo de justicia restaurativa tanto en el sistema mixto como en el Sistema Acusatorio. No sólo se persigue al agresor, sino que también se apoya a la víctima y a su núcleo familiar para superar las secuelas de una agre- 
sión". De esta manera, se asume que "la justicia restaurativa permite el restablecimiento pleno de los derechos de las víctimas porque se detiene la violencia, se aleja al agresor y se brindan medidas de protección" (Fiscalía General de la Nación).

Sin embargo, la alternativa que hoy aporta el CAVIF continúa sin adecuarse a los planteamientos y principios de la Justicia Restaurativa (más próxima a la figura de la conciliación que establecía la Ley 600 de 2000), aun cuando contemple una serie de recursos (talleres, seminarios, manejo terapéutico y seguimiento) para brindar una atención más integral a su población objetivo. De allí, es posible afirmar que aún no se ha hecho efectiva la implementación de programas de Justicia Restaurativa en los términos que establece la Ley 906 de 2004 (Artículos 518 a 527), debido probablemente a la adopción reciente del paradigma y al carácter novedoso del mecanismo, así como a la falta de procesos pedagógicos dirigidos a los funcionarios a quienes corresponde su implementación.

Con el fin de acompañar la puesta en marcha de estos procesos de Justicia Restaurativa, se ha diseñado una investigación para elaborar los criterios de un programa piloto de justicia restaurativa para casos de violencia intrafamiliar en el CAVIF, con el fin último de apoyar la implementación efectiva del mecanismo. A través de esta investigación se busca favorecer la puesta en marcha de programas de Justicia Restaurativa para casos de violencia intrafamiliar, cuyos insumos permitan extender su aplicación a escenarios jurídicos en los que se tramitan diversos conflictos.

\section{La violencia doméstica}

Para pensar en la violencia doméstica se requiere hacer un previo análisis del comportamiento violento, que para algunos estudiosos de la materia puede ser entendido como una condición inherente a todos los seres humanos, que su expresión depende de múltiples factores y contextos. La violencia puede ser interpretada como un acto reprobable, aceptable o relativo para calificar de acuerdo con el juicio argumentativo del contexto, la situación y el significado que cultural, social, familiar o individual se le otorgue a determinada conducta. La violencia puede ser aceptada y justificada con diversos razonamientos, ya sea desde una ideología política predominante, un momento histórico, un paradigma de conocimiento científico que estaría de acuerdo con un sistema de creencias, normas y valores aceptado en un determinado grupo humano.

La violencia para algunas corrientes del conocimiento se adquiere y se aprende a lo largo de un proceso, con una importante influencia en las representaciones sociales y culturales donde al comportamiento violento se le asocia generalmente con el abandono, las vejaciones, la negligencia, el maltrato físico, el maltrato psicológico, el daño moral, la discriminación, la marginación, entre otros calificativos, producto de determinados ambientes basados en la competitividad, el dominio, la posesión, la fuerza, y concepciones de éxito basados en la riqueza y el poder (Beceiro, 2000; Clemente \& Espinosa, 2001).

La violencia, analizada desde el modelo explicativo del aprendizaje social de Bandura (1973), por ejemplo, estaría asociada a la explicación del comportamiento violento que se aprende y mantiene por medio de experiencias ambientales, de manera directa o vicaria; en contraste, el aprendizaje de la agresión es controlado por experiencias de reforzamiento y castigo como el aprendizaje de cualquier otra conducta, es decir, que los niños y las niñas aprenden de los adultos y de sus pares a través de dos fenómenos básicos en su teoría: la observación y la imitación. Además, otros autores como (Pueyo \& Redondo 2007), consideran que en el aprendizaje del comportamiento violento intervienen mecanismos interrelacionados como: la asociación diferencial con personas que muestran hábitos y actitudes violentas, la adquisición por el individuo de definiciones favorables a la trasgresión de las normas, el reforzamiento diferencial de sus comportamientos violentos y la imitación de modelos que favorecen la violencia.

Otros estudios, siguiendo los hallazgos del modelo del aprendizaje, profundizan respecto al modo en que el sujeto percibe e interpreta los aconte- 
cimientos y la forma en que responderá: violenta o con algún otro comportamiento. Asimismo, Bandura (1986) introdujo el concepto de autoeficacia, el cual hace especial énfasis en lo competente que se siente el sujeto al responder de manera violenta o no. Estos planteamientos teóricos comparten la tesis de que la cognición y sus procesos psicológicos de atención, percepción, memoria, emociones y aprendizaje en la niñez tienen una función relevante en el mantenimiento de la estabilidad o la modificación del comportamiento violento a lo largo del tiempo, así como de una situación a otra, en las que pueden influir las variables contextuales en la familia (condiciones socioeconómicas, prácticas de crianza de los padres, experiencias vitales estresantes, consumo de drogas, alcohol, por parte de los padres) y fuera de ella (violencia en el barrio, la pobreza, relación con compañeros, exposición a la violencia a través de los medios de comunicación). Estas variables contextuales ayudan a moldear la cognición, la cual después ejerce una influencia más directa en el comportamiento agresivo (Eron, Walder \& Lefkowitz, 1971).

La violencia tiene importantes diferencias con la peligrosidad y la agresión, esta última tiene un nivel de análisis desde la etología, la psicofisiología, neurobiología, lo que implica que se presenta la agresión como la capacidad del organismo para defenderse de los peligros potenciales procedentes del exterior y debe analizarse, como la respuesta adaptativa bajo impulsos e instintos que responden a estímulos específicos desencadenantes al servicio del hombre, que ayuda a la adaptación y supervivencia en el medio en que vive, (Lorenz, 1976; Clemente \& Espinosa, 2001). Es decir, que un organismo responde de determinada manera a una situación en particular en función del estado en que se encuentre en dicho momento. Dicho estado sólo puede estar contenido como especificaciones físicas de las células que constituyen al organismo, sobre todo, como especificaciones del sistema endocrino. Así, podemos afirmar que, tanto factores genéticos como ambientales y las interacciones entre estas deben intervenir para alterar la base fisiológica del comportamiento Gray, 1992.
Los estudios encaminados a la predisposición a la agresión y su relación con el comportamiento violento, exige al parecer integrar los resultados de los campos fisiológicos, neuroendocrino, psico-fisiológicos, conductual, cognitivo, emocional y de la personalidad. A medida que aumente el conocimiento en cada uno de estos campos, aumentará la necesidad de instrumentos heurísticos para conceptuar estas influencias diversas y sus interacciones (Newman, Kosso \& Patterson, 2002).

En este orden de ideas autores como Pueyo \& Redondo (2007), llaman la atención sobre la importancia de definir correctamente la violencia, encontrando una doble interpretación en tanto acción (comportamiento) y disposición (capacidad y atributo psicológico). Aluden a la necesidad de diferenciar la cualidad de ser violento, que puede entenderse a la vez como sinónimo de peligrosidad y la acción de comportarse violentamente. Estos mismos autores consideran que la violencia es una estrategia psicológica para alcanzar un fin determinado, por tanto, identifican que el agente "causal del acto violento es el individuo, pero éste actúa en un contexto o situación que facilita o estimula la aparición" (Pueyo \& Redondo, 2007, p. 157). De esta forma se identifican componentes que modulan y mantienen la conducta violenta, sustentan que los determinantes de la violencia como estrategia no son los mismos que los de la acción violenta, sino que esta última es el determinante más importante en la decisión intencionada de actuar de ese modo.

Para complejizar y profundizar la definición de la violencia y para acceder a un estudio riguroso (Pueyo \& Redondo, 2007) distinguen cinco propiedades, a saber: 1) la complejidad: entendida como estrategia psicológica en la que intervienen aspectos cognitivos; 2) actitudinales, emocionales y motivacionales; 3) es heterogénea: por presentar varios tipos de violencia (física, psicológica, sexual, deprivación, abandono, económica, entre otros). Además pudiendo ser auto-dirigida, interpersonal y colectiva; 4) es multicausal, es decir, que coinciden en el acto numerosos factores de riesgo; 5) es infrecuente sobre todo en el tipo de violencia grave y muy grave. 
En este orden de ideas, cada tipo de violencia tiene factores de riesgo y de protección específicos, y por tanto se deben adecuar los procedimientos genéricos de predicción de riesgo al tipo concreto de violencia y, por ende, de los protocolos diseñados para cada caso. Es importante hacer anotar que la valoración del riesgo tiene las siguientes ventajas: a) el constructo que se estudia es continuo, variable y específico, lo que permite hacer pronósticos; b) tiene la posibilidad de la intervención individual y contextual; c) puede distinguir entre los factores estáticos y dinámicos que son susceptibles de modificar o no.

En este orden de ideas, los programas de intervención con agresores de violencia intrafamiliar han demostrado diversos grados de efectividad. Pocos de ellos han demostrado resultados duraderos y cambios en las dinámicas de relación familiar. La pena privativa de la libertad no ha resultado efectiva y ha ocasionado, por el contrario, rupturas en el tejido social. Adicionalmente, el sistema penal retributivo continúa centrado en la sanción punitiva al infractor, desconociendo las necesidades de las víctimas y las posibilidades de reparación del daño causado por el delito, tanto a la familia como a la comunidad. Por estas razones, el delito de violencia intrafamiliar ha comenzado a ser abordado a través de diversos programas de justicia restaurativa, principalmente, del sistema de mediación víctima-infractor. Por esto, a continuación se presenta una breve caracterización de la violencia intrafamiliar y de las formas de intervención.

Existen diversas nociones de violencia intrafamiliar (VIF), todas coinciden en que está referida a la pareja exclusivamente, y particularmente a la agresión física, el maltrato psicológico y las relaciones sexuales forzadas $u$ otras formas de coacción sexual y diversos comportamientos dominantes (como el aislamiento del entorno social, la vigilancia o la restricción en el acceso a la información, la asistencia o los recursos económicos, entre otros) que ejerce el hombre (marido, pareja o ex pareja) sobre la mujer (Heise \& García-Moreno, 2003 citados por Echeburúa, 2004). Tal como señala Walker (1991) la VIF es un patrón de conductas abusivas utilizado por una persona en una relación íntima contra otra para ganar poder, control y autoridad. Al ocurrir en un espacio privado, no sólo pone en riesgo la seguridad y bienestar de la mujer, sino también de quienes conforman la familia, que se entiende como el grupo de personas que viven bajo el mismo techo y permanecen en continua interacción (Medina, Aracena \& Bilbao, 2004; Traverso, 2007). Según los porcentajes para Colombia, más del $91 \%$ de las víctimas de maltrato conyugal son mujeres (Ribero \& Sánchez, 2004).

La familia es el primer agente socializador y sobre ella recaen las responsabilidades de educación, protección y crianza de los hijos, por tanto juega un importante papel en el crecimiento, desarrollo y bienestar del individuo (Echeburúa, Paz, Fernández-Montalvo, Amor, 2004), es allí, paradójicamente, donde se mezclan los sentimientos más amorosos y a su vez los más destructivos. También es el lugar en el que se satisfacen las necesidades más básicas del ser humano, e igualmente sitio de mayor riesgo para las conductas violentas (Ballanga et ál., 2008).

Es en el hogar donde se concentra el mayor índice de violencia en nuestra sociedad. $Y$ a pesar de que actualmente las denuncias han aumentado, en parte porque se ha logrado visibilizar dicho fenómeno, es aún mayor el número de casos que no se denuncian, debido a que se convierte en una dinámica reiterada de relación para muchas familias. Adicionalmente, el lazo afectivo que los une facilita que se toleren, acepten y hasta habitúen a dinámicas violentas de relación. No por ello deja de ser preocupante que dicho fenómeno permanezca impune; sin embargo, se ha venido generando mayor conciencia en que la mujer no debe aceptar la violencia y tampoco ocultarla (Soria \& Rodríguez, 2003; Madina, 2005).

La VIF se caracteriza por la desigualdad de poder en la relación, la continuidad en el tiempo y la sensación de la mujer, al no poder evadirse de la relación por ver amenazada su vida (Madina, 2005). Igualmente, el constante desequilibrio en las decisiones que se toman con respecto al hogar, donde el hombre impone qué debe hacerse y cómo debe hacerse, facilita la agresión, sobre 
todo cuando existe oposición por parte del dominado. Estas situaciones de desigualdad facilitan pasar del conflicto inherente a los seres humanos a la violencia, debido a la incapacidad o dificultad para comunicar las diferencias y aceptarlas, lo que lleva al empleo de la fuerza y la agresión como forma de solucionar los problemas (Traverso, 2007).

Sin embargo, la VIF no sólo es física, si esto se entiende como todo acto que está encaminado a dejar secuelas en el cuerpo de la persona (Ballanga et ál., 2008). La violencia psicológica es quizá la que genera más efectos adversos sobre quien se ejerce, haciéndose compleja su detección. Implica la humillación constante, la desvalorización, el control de la otra persona (incluso a nivel económico), amenazas y todo acto que tienda a denigrar al ser humano (Madina, 2005). En muchos hogares la prevalencia de una de estas formas de maltrato se visualiza en las secuelas que deja a sus víctimas, siendo variable su aparición. La probabilidad de que la víctima experimente diferentes tipos de maltrato simultáneamente es muy alta. Usualmente, la mujer denuncia sólo un tipo de maltrato, que suele ser el físico, ocultándose el maltrato psicológico y el sexual que quizá suelen ocurrir con mucha frecuencia. Este es uno de los mayores limitantes para tener cifras reales de dicha problemática (Ribero \& Sánchez, 2004).

La VIF puede convertirse en un método efectivo para que el maltratador consiga sus objetivos, los cuales están relacionados con el poder y el control sobre la pareja. El maltratador se percibe a sí mismo como una persona que posee las características físicas, las capacidades mentales, y el derecho a manejar la vida de otros, razón por la cual busca descargar sus sentimientos de ira y agresión frente a su pareja a quien considera vulnerable, y por tanto subordinada (Jennings \& Murphy, 2000).

El maltratador que cree erróneamente poseer el control y dominio sobre su pareja, le niega la posibilidad de decisión, asume su dirección y no acepta que puedan tener los mismos derechos. Ésta es una de las tantas distorsiones que tiene el hombre que maltrata a su pareja. Igualmente, considera que la mujer debe obedecer órdenes y llevar una vida sumisa, es una forma de copiar modelos que culturalmente ha vivido o que ejercían sus familias de origen (Ehrensaft et al., 2007, Soria \& Rodríguez, 2003).

A pesar de que actualmente la mujer ha asumido un nuevo rol en la sociedad, las cifras de VIF aumentan año tras año. El hecho de que se le reconozca igualdad de condiciones que al hombre, específicamente a nivel laboral, le permite a ésta asumir una actitud de control y decisión sobre su propia vida. En este sentido, la mujer ya no depende económicamente del hombre, situación que pone a este último en una posición de competitividad y descontrol. Sin embargo, en muchos casos la dependencia es afectiva, razón por la cual no se rompe el vínculo con el maltratador, a pesar de vivir estos hechos de violencia, de esta forma las víctimas pueden sentirse incapaces de escapar del control de los agresores al estar sujetas a ellos por coacciones y amenazas graves (Corral, 2000 citado por Echeburúa et al., 2004).

La literatura indica que un alto porcentaje de maltratadores provienen de familias disfuncionales en las que se ejercía la violencia, ya fuera contra ellos o entre sus padres. Usualmente, se encuentra que fueron tanto observadores como receptores directos de violencia y malos tratos (Ruiz \& Expósito, 2008). Para el caso de los maltratadores que van a prisión, se encuentra que más de la mitad, fueron víctimas de abusos y malos tratos en su infancia (Soria \& Rodríguez, 2003).

De acuerdo con los estudios revisados, se encuentra que es muy alto el porcentaje de mujeres maltratadas que continúan con la relación de pareja, razón por la cual el maltratador sigue cometiendo actos de violencia contra ellas. Tales dinámicas se vuelven cíclicas y cada vez más frecuentes. Una vez se presenta el primer hecho de violencia y a pesar del arrepentimiento del agresor, la probabilidad de continuar en actos violentos es alta, ya que el agresor minimiza los efectos de sus actos y el agredido justifica dicho comportamiento (Echeburúa, 2004, Madina, 2005). Estos mismos autores describen la fase de incremento de la tensión que se caracteriza por un aumento de ansiedad y 
alteración y que puede ir acompañada de agresión física en los casos más graves. Luego viene la descarga de violencia caracterizada por un desfogue de tensión, acompañada de agresión física y sensación de bienestar para el maltratador, ya que con esto consigue sus objetivos de control, después viene una fase de arrepentimiento en la que el hombre trata de recuperar el equilibrio de la relación por medio de justificaciones, manifestaciones de cariño, promesas de no recaer, todo con el objetivo de conciliar y seguir con su pareja.

La tendencia es la desaparición de la fase de arrepentimiento y por tanto a que la descarga de violencia sea más frecuente (Ortiz, 2005). Los rasgos de personalidad del maltratador tienden a ser complementarios con los de la víctima. De acuerdo con los estudios, usualmente ambos tienen historial de maltrato en sus hogares de origen, por lo cual la dinámica de relación violenta se perpetúa y no es considerada por la víctima como tal (Fariña, Arce \& Novo, 2008). Lo que se puede decir en cuanto al perfil del maltratador es que presenta alteraciones psicológicas en el ámbito del control de la ira, de la empatía y expresión de emociones, de las cogniciones sobre la mujer y la relación de pareja, de las habilidades de comunicación y de solución de problemas (Echeburúa, 2004).

Respecto a la intervención preventiva con los agresores, gran número de programas para prevenir la VIF están enfocados a trabajar con las víctimas, pero muy pocos con los victimarios. Una de las razones que se encuentra para ello es que las víctimas, que en su gran mayoría son mujeres, perciben como problemática la situación de abuso y surge la necesidad de buscar apoyo y ayuda a un tercero, contrario a lo que sucede con los victimarios, quienes en muy pocos casos perciben como problemática la situación de violencia y menos aún su comportamiento (Chambersb, Ecclestona, Dayb, Wardc \& Howellsb, 2008). Aún así, se recomienda el tratamiento para el agresor, pues muchas mujeres continúan viviendo con éstos a corto o largo plazo, o en su defecto, el maltratador buscará otras víctimas. Por tanto, intervenir directamente sobre el generador de la violencia sería una forma de prevenir daño hacia otras mujeres (Echeberúa et al., 2004). En la medida en que se pueda trabajar con los maltratadores disminuirá también la violencia, a pesar de lo difícil que resulta cuando no hay voluntad de cambio por parte del agresor (Echeburúa, 2004).

Respecto al tratamiento con maltratadores en prisión, las medidas penales para los maltratadores no tienen una función rehabilitadora, tiene como función aislar al individuo violento del foco de agresión y de una manera directa proteger a la víctima; sin embargo, son pocos los casos en los que se reeduca para que éstos cambien realmente su comportamiento (Garrido \& Echeburúa et al., 2006).

A pesar de que en Colombia los índices de VIF son muy altos, y que igualmente han surgido programas de prevención e intervención frente a esta problemática, son muy pocos los estudios que se han hecho para evaluar impacto y efectividad. Igualmente los estudios que se han hecho tienen muchas limitaciones en cuanto a evaluar cada uno de los tipos de violencia: física, sexual y psicológica. Adicionalmente, los contextos que han sido evaluados difieren notablemente. No es lo mismo evaluar una población urbana que una rural, o una carcelaria. En este sentido, hallar un consenso en cuanto a los programas que se han trabajado con población maltratadora es muy difícil. Sin embargo, en países como España, Guatemala, Chile, entre otros, estos estudios tienden a dar cuenta de dicho fenómeno de una manera más amplia que en el nuestro.

Es común que se preste atención y apoyo a las víctimas directas del maltrato, tratando de empoderar a la mujer para prevenir que se continúe con los ciclos de violencia. Sin embargo, se ha observado que a pesar de este esfuerzo, algunas mujeres deciden volver con sus parejas o en su defecto se retractan de las denuncias hechas, puesto que muchas lo hacen como una forma de "amenaza” (Fariña et ál., 2008). Las mujeres que se retractan, en parte lo hacen por las consecuencias negativas que ello trae para sus parejas. El hecho de que estos puedan ir a prisión y que no aporten económicamente al hogar, no les permite continuar con el proceso. Otras prefieren pensar 
que sus parejas van a cambiar su comportamiento y otras sencillamente requieren de estas dinámicas. Sin embargo, tratar psicológicamente a la víctima y prescindir de la ayuda al agresor es, a todas luces, insuficiente (Ortiz, 2005).

También son muy pocos los programas que le apuestan a trabajar con los maltratadores. Una de las principales razones tiene que ver con la alta tasa de abandonos por parte de los agresores, puesto que sus características de personalidad, sus distorsiones cognitivas, patrones de relación aprendidos desde el hogar, la influencia cultural, entre otros, no favorecen la adhesión al tratamiento (Echeburúa, 2004; Ruiz \& Expósito, 2008). A su vez, porque no reconocen que otro diferente a él mismo tenga el control de las situaciones, se perciben a sí mismos como autosuficientes y consideran que no requieren del apoyo de nadie (Medina et al., 2004).

A pesar de ello, los tratamientos psicológicos con maltratadores ofrecen resultados satisfactorios, pues se ha logrado reducir las conductas de maltrato y evitar la reincidencia, así como un mayor bienestar para el agresor y para la víctima (Corsi, 1996, Echeburúa \& Fernández-Montalvo, 1997 citados en Echeburúa et ál., 2004). En estudios realizados en prisión, se observa que la posibilidad de cambio de la conducta violenta se mide en función de la responsabilidad que asume el maltratador por su conducta. Cuando el maltratador es consciente de que ha causado daño y sufrimiento a su víctima es más fácil ayudarle a tener control sobre su comportamiento. Entre tanto, un sujeto que se muestre menos dispuesto a un cambio de conducta y a aceptar su responsabilidad por el daño causado a la víctima, tiene menos probabilidad de mejorar su comportamiento y la probabilidad de recaer por el mismo problema es alta. Estos sujetos tienen una visión muy negativa de sí mismos, de sus parejas, del terapeuta y en general de las personas con quienes interactúan (Rodríguez, Azcárate, Echauri \& Martínez, 2008, Lila, Herrero \& Gracia, 2008; Chambersb et al., 2008).

Frente a las orientaciones teóricas de la intervención de acuerdo con estudios consultados sobre intervención con maltratadores, los dos enfoques de mayor impacto son la terapia en reeducación y la terapia cognitivo-conductual.

Los programas de intervención que están encaminados a la reeducación tienen por objetivo educar al maltratador y brindarle herramientas para que éste aprenda a manejar su comportamiento agresivo. Implican evaluación de aspectos que podrían interferir con la terapia (aptitudes, factores de riego y protección, creencias, actitudes sexistas, estado clínico, comportamientos adictivos, adherencia o no al tratamiento, entre otros), esto con el fin de ajustar el tratamiento de acuerdo con su perfil y poder observar evolución y cambio. Se trabaja por medio de charlas y conferencias en temas relacionados con la VIF, como son su explicación conceptual, las manifestaciones del comportamiento agresivo, consecuencias para las víctimas, precipitantes, factores de riesgo, entre otros. Asimismo, se enfocan en enseñarle habilidades de afrontamiento, manejo de emociones, control de celos, entrenamiento en habilidades sociales, un auto-examen a su conducta para evaluar el patrón de socialización en relación con la pareja (Labrador, Paz, \& Fernández, 2008). Se ha empleado el tiempo fuera, como mecanismo de reconocimiento de emociones y con ello de control de impulsos (Ortiz, 2005).

Algunos de estos programas de intervención han logrado que el maltratador cambie el modo de agredir y pase de la violencia física a la psicológica; sin embargo, esto se debe en parte a que la violencia psicológica es menos visible, pero aún es muy preocupante debido a que puede generar mayores secuelas psicológicas para la víctima (Ortiz, 2005). Este tipo de intervención, a pesar de que ha tenido efectos positivos para algunos casos, trae serias limitaciones, ya que los maltratadores utilizan la información para justificar su comportamiento o para explicar a la víctima porqué lo hacen (Lila et ál., 2008). En este sentido, muestran que más allá de un cambio a nivel conductual generan un cambio a nivel actitudinal (Ruiz \& Expósito, 2008).

Los programas de tratamiento que han mostrado efectividad están dirigidos a modificar las distor- 
siones cognitivas de los maltratadores. Esto se ha logrado específicamente con sujetos que reconocen y asumen su responsabilidad en la conducta violenta y quienes desean un cambio en su comportamiento. Para los casos en que estas variables no se encuentran presentes es muy difícil lograr cambios de comportamiento (Arias \& Expósito, 2008; Lila et ál., 2008; Smith, 2007; Chambersb et al., 2008; Madina, 2005 y Echeburúa et al., 2004). Además, de acuerdo con los estudios, a pesar de que los programas de mayor duración tienen una alta probabilidad de cambio, éste sólo se logra en un porcentaje mínimo de maltratadores (Echeburúa, 2004; Lila et ál., 2008), a pesar de que están enfocados al manejo de las distorsiones cognitivas, tienen un enfoque grupal, se hacen seguimientos periódicos, y los pacientes acuden voluntariamente.

Por tanto, asistir voluntariamente y con propósito de cambio ha sido uno de los motivos por los cuales los maltratadores abandonan el tratamiento, sumado a sus características de personalidad que no favorecen en mucho la adherencia al mismo (Ferrer, Bosch, García, Manassero \& Gili, 2004). Además, para estos casos los sesgos cognitivos que posee el maltratador son tan arraigados que tienden a responsabilizar siempre a la pareja, o a minimizar y negar sus actos, por lo cual la terapia que busca responsabilizarlos de sus actos y manejar sus distorsiones no es un atractivo para ellos (Chambersb et ál., 2008). En este sentido, los programas son efectivos sólo para un reducido número de casos y en la mayoría se cambian las tácticas o modos de agredir, pero permanece la intención de hacer daño.

Ahora para medir la efectividad de los programas de intervención, de acuerdo con estudios realizados, se presenta en general una alta efectividad en aquellos casos en los que se completa todo el proceso y el maltratador asiste voluntariamente. En Colombia, los estudios sobre VIF han sido escasos y los que han centrado su interés en esta problemática, aún tienen dificultades para medir incidencia, evaluar el grado de severidad del maltrato y principalmente para evaluar cada uno de los tipos de violencia en el hogar. La atención se ha centrado en la prevención, a través de campa- ñas educativas y talleres de sensibilización, pero muchas de estas acciones no son evaluadas, por tanto, no permiten medir efectividad e impacto (Ribero \& Sánchez, 2004). Igualmente, aún no hay un consenso institucional que permita tener cifras exactas y reales de esta problemática y menos para los casos que han sido evaluados en cárceles. Sin embargo, tener cifras nos permite decir qué tanto afecta esta problemática a una sociedad y sobre todo para aportar soluciones que generen un cambio social (Traverso, 2007).

\section{La Justicia Restaurativa. Marco teórico y normativo}

En Colombia la implementación de programas de Justicia Restaurativa se ha venido haciendo efectiva a partir de la normatividad de base, la cual define y establece las nuevas formas de aplicación de justicia. De manera expresa, el término Justicia Restaurativa fue consagrado con la aprobación del Acto Legislativo 03 de 2002 en el que se establece que la Fiscalía General de la Nación, en ejercicio de sus funciones, debe "velar por la protección de las víctimas, los jurados, los testigos y demás intervinientes en el proceso penal. La ley fijará los términos en que podrán intervenir las víctimas en el proceso penal y los mecanismos de justicia restaurativa" (Artículo 250, numeral 7).

Posteriormente, esta disposición fue desarrollada en la Ley 906 de 2004 que expidió el nuevo Código de Procedimiento Penal, en la que se define la Justicia Restaurativa como,

(...) todo proceso en el que la víctima y el imputado, acusado o sentenciado participan conjuntamente de forma activa en la resolución de cuestiones derivadas del delito en busca de un resultado restaurativo, con o sin la participación de un facilitador. Se entiende por resultado restaurativo el acuerdo encaminado a atender las necesidades y responsabilidades individuales y colectivas de las partes y a lograr la reintegración de la víctima y del infractor en la comunidad, en busca de la reparación, la restitución y el servicio a la comunidad (Artículo 518). 
Esta Ley contempla tres mecanismos de Justicia Restaurativa, a saber: la conciliación preprocesal, la conciliación en el incidente de reparación integral y la mediación (Artículo 521), así como seis reglas generales (Artículo 520) para la implementación de los programas de justicia restaurativa: 1) Consentimiento libre y voluntario de la víctima y el imputado, acusado o sentenciado de someter el conflicto a un proceso restaurativo. 2) Los acuerdos que se alcancen deberán contener obligaciones razonables y proporcionadas con el daño ocasionado con el delito. 3) La participación del imputado, acusado o sentenciado no se utilizará como prueba de admisión de culpabilidad en procedimientos jurídicos ulteriores. 4) El incumplimiento de un acuerdo no deberá utilizarse como fundamento para una condena o para la agravación de la pena. 5) Los facilitadores deben desempeñar sus funciones de manera imparcial y velarán porque la víctima y el imputado, acusado o sentenciado actúen con mutuo respeto y 6) La víctima y el imputado, acusado o sentenciado tendrán derecho a consultar a un abogado.

En el contexto de familia, la Ley 906 de 2002 contempla la violencia intrafamiliar como uno de los delitos que requieren querella (Artículo 229) ${ }^{1}$. Sin embargo, la aplicación del principio de oportunidad ${ }^{2}$ abre a los sujetos activos y pasivos involucrados en un delito de familia, la posibilidad de acordar la reparación civil y llegar a un acuerdo antes de iniciar un proceso judicial penal. Para este efecto, el fiscal puede acudir a los mecanismos de suspensión del procedimiento a prueba o interrupción de la actuación penal con el fin de verificar el cumplimiento de las condiciones convenidas para dar aplicación a la renuncia y dar solución al caso por la vía de la Justicia Restaurativa.

1 Este Artículo ha sido reformado por el Artículo 33 de la Ley $1142 / 2007$ en lo referente a las penas.

2 El principio de oportunidad "es la facultad constitucional que le permite a la Fiscalía General de la Nación, no obstante que existe fundamento para adelantar la persecución penal, suspenderla, interrumpirla o renunciar a ella, por razones de política criminal, según las causales taxativamente definidas en la ley, con sujeción a la reglamentación expedida por el Fiscal General de la Nación y sometido a control posterior de legalidad ante el juez de garantías (...). Por medio de la aplicación del principio de oportunidad, el Estado finalmente renuncia a investigar una conducta con características de delito o a la acusación de los presuntos responsables (...)" (Fiscalía General de la Nación, 2005, p. 167).
La Justicia Restaurativa es un paradigma que confronta los sistemas tradicionales (punitivo y rehabilitador), a la vez que promueve la reparación del daño causado por el delito. Hace énfasis en la dimensión del perjuicio antes que en la dimensión de la trasgresión de una norma jurídica (sistema punitivo) o de las necesidades del infractor (sistema rehabilitador), con lo cual reconoce que "la función principal de la reacción social no es ni castigar, ni tratar, sino crear las condiciones para que una reparación \& una compensación razonable de los perjuicios se puedan realizar" Walgrave (1999, p. 9).

La Confraternidad Carcelaria Internacional (2005) define la Justicia Restaurativa como una teoría de justicia que enfatiza en la reparación del daño causado o presentado por una conducta delictiva, cuyos objetivos se alcanzan a través de procesos cooperativos que incluyan a todos los interesados. En esta medida, se destaca por la participación activa de las partes involucradas, con el fin de que sean ellas mismas quienes propongan las estrategias de justicia frente al conflicto. A diferencia de los sistemas tradicionales -retributivo y rehabilitador- la Justicia Restaurativa propende por la responsabilidad del infractor, la reparación de la víctima y la participación de la comunidad. De esta manera, pretende afrontar los perjuicios sociales, psicológicos y relacionales, trascendiendo la mera respuesta a los daños materiales y físicos causados por la conducta delictiva. Bajo esta perspectiva victimológica, la ambición maximalista de la justicia restaurativa obliga a concebir una relación coherente entre las partes, los procesos y las prácticas restaurativas, por una parte, y el Estado Social de Derecho y sus instituciones, por otra parte (Berinstain 1998), puesto que ambos tienen misiones complementarias en la reparación de las consecuencias de un delito otorgando a la justicia restaurativa, prioridad a las respuestas provenientes del exterior del sistema judicial y busca vías alternas para promover la primacía de la comunidad, esto no implica la exclusión de sanciones impuestas desde el aparato judicial. En consecuencia, Berinstain (1998) resume los rasgos fundamentales del modelo restaurativo, en comparación con el modelo retributivo, en los siguientes aspectos: 
Tabla 1. Resumen de rasgos del modelo restaurativo, según Berinstain (1998)

\begin{tabular}{cc}
\hline MODELO RETRIBUTIVO & MODELO \\
RESTAURATIVO
\end{tabular}

El delito es la infracción a El delito es la acción que la norma penal del Estado. causa daño a otra persona.

Se centra en el reproche, Se centra en la solución la culpabilidad, mirando al del problema, en la respasado, a lo que el delin- ponsabilidad y obligaciocuente hizo. nes mirando al futuro.

Se reconoce una relación Se establece un diálogo de contrarios, de adversa- y una negociación norrios, que vencen y someten mativa que imponga al al enemigo en un proceso delincuente una sanción normativo legal.

restauradora.

La solución del conflic-

El castigo es la consecuen- to está en la reparación cia natural, dolorosa, que como un medio de restambién conlleva o preten- taurar ambas partes, de la prevención general y víctima y delincuente. especial. Tiene como meta la reconciliación.

El delito se percibe como un conflicto (ataque) del El delito se reconoce individuo contra el Estado. como un conflicto interSe menosprecia su dimen- personal. Se reconoce el sión interpersonal y con- valor del conflicto.

flictiva.

El daño que padece el sujeto pasivo del delito se compensa con (reclama) otro daño al delincuente.

Se pretende lograr la restauración del daño social.

Se margina a la comunidad La comunidad como ca(y a las víctimas) y se la talizador de un proceso ubica de forma abstracta restaurativo versus el en el Estado. pasado.

Se promueve (Madina, 2005), se fomenta el talan- Se incentiva la reciprote competitivo y los valores cidad. individuales.

La sanción es la reacción del Estado contra el delincuente. Se ignora a la víctima y el delincuente permanece pasivo.
Se reconoce el papel de la víctima y el papel del delincuente, tanto en el problema (delito) como en su solución. Se reconocen las necesidades y los derechos de la víctima. Se anima al delincuente a responsabilizarse.

\begin{tabular}{ll}
\hline \multirow{2}{*}{ MODELO RETRIBUTIVO } & \multicolumn{1}{c}{$\begin{array}{c}\text { MODELO } \\
\text { RESTAURATIVO }\end{array}$} \\
\hline & $\begin{array}{l}\text { Se define la responsa- } \\
\text { bilidad del delincuente }\end{array}$ \\
El deber del delincuente es como la comprensión & col impacto de su acción \\
cumplir (sufrir) la pena. & $\begin{array}{l}\text { y el compromiso de re- } \\
\text { parar el daño. }\end{array}$
\end{tabular}

El delincuente no tiene res- El delincuente tiene responsabilidad en la solución ponsabilidad en la soludel problema. ción del conflicto.

El delito se define a tenor de la formulación legal, sin El delito se entiende en tomar en consideración las todo su contexto moral, dimensiones morales, so- social, económico y pociales, económicas y polí- lítico.

ticas.

El delincuente tiene una Se reconoce a la víctima deuda con el Estado y la la deuda/responsabilisociedad en abstracto. dad.

El estigma del delito El estigma del delito es im- puede borrarse por la borrable acción reparadora/restauradora.

No se fomenta el arrepen- Se procura el arrepentitimiento, el perdón. miento y el perdón.

La justicia está exclusiva- La respuesta al delito se mente en manos de profe- crea desde los propios sionales gubernamentales. protagonistas.

De acuerdo con García-Pablos (1999), a través del modelo de justicia restaurativa se busca un modelo integrador de respuesta al fenómeno social del delito que ofrezca una imagen de justicia más humana; una justicia más legal que profesional, próxima al ciudadano, de marcado perfil comunitario, pacificadora, comunicativa e integradora. Una justicia que comprenda los conflictos desde dentro y trate de buscar soluciones a los mismos sin imponerlas. Constructiva y no represiva. Que se sintonice con los valores éticos, con el sentido común del ciudadano, con la experiencia humana y comunitaria, sin refugiarse en formalismos y exigencias utilitaristas. Todo ello, mediante procedimientos des-formalizados, flexibles y operativos que faciliten la negociación, el tratamiento del conflicto y su solución satisfactoria sin perjuicio del control, y garanticen, por supuesto, los derechos fundamentales de los implicados.

La investigación y experiencia internacional aportan un marco teórico y práctico fundamental para avanzar en el diseño de programas de justicia 
restaurativa y en la aplicación de sus principios, así como para establecer criterios de viabilidad y condiciones para la aplicabilidad en distintos escenarios de la administración de justicia. El amplio desarrollo internacional que ha tenido la justicia restaurativa en los últimos treinta años, ha generado una serie de documentos jurídicos que promueven y regulan la implementación de los diferentes mecanismos y programas en delitos de diversa gravedad. El entendimiento de que la justicia restaurativa ha pasado a ser una importante alternativa a los procesos de enjuiciamiento en la justicia penal ha favorecido la implementación de programas en sistemas de justicia altamente variados.

El auge de los programas alternativos de administración de justicia en el campo de familia, ha obedecido a la necesidad de desjudicializar y descriminalizar los conflictos familiares que admitan vías alternas de tramitación. La importancia otorgada a los procesos restaurativos en este campo ha radicado en el estatus otorgado a la familia como institución socializadora de base que, por dinámicas propias o mediante facilitadores, genera mecanismos de concertación que incidan positivamente en las relaciones interpersonales y prevengan desórdenes en el grupo social y formas violentas o delictivas de relación. En este sentido, la violencia intrafamiliar ha sido identificada como un factor de riesgo desde una perspectiva de prevención de la criminalidad urbana, por lo cual los gobiernos han priorizado estrategias que permitan hacer frente a la dimensión pública del conflicto y contrarrestar el resquebrajamiento de la convivencia.

\section{Método}

El estudio obedeció a un diseño de tipo descriptivo exploratorio. Para la recolección de la información se empleó una metodología de tipo cualitativo.

\section{Muestra}

Se contó con una muestra intencional integrada por veinte usuarias que asistieron al CAVIF por situaciones de violencia intrafamiliar y 18 funcionarios, entre fiscales, técnicos judiciales, secretarios judiciales, asistentes judiciales y asistentes de fiscal, empleados del CAVIF en el momento de la investigación. Las usuarias entrevistadas fueron, en todos los casos, denunciantes de violencia intrafamiliar.

\section{Instrumento}

La recolección de información se realizó a través de dos entrevistas a profundidad (usuarios y fiscales) diseñadas específicamente para el estudio. El análisis de los datos cualitativos se realizó con el programa SPADT (para análisis de texto). Los resultados arrojados por SPADT se presentan en una tabla con un listado de palabras que no se incluyen aquí, ordenadas por su frecuencia de uso; este listado es el resultado de una depuración en la cual se eliminaron conectores (el, lo, las, etc.) y palabras que no ofrecían información para el trabajo (hacen, desde, todas, propias, etc.). Por último, se efectuó un proceso de equivalencia de palabras, tomando como criterio las raíces de las mismas para realizar las paridades.

\section{Resultados}

Los funcionarios del CAVIF consideran que la violencia intrafamiliar es un problema fundamentalmente cultural y social en el que intervienen, además, factores como: lo económico, la condición social y la educación. Afirman que este delito les genera cuestionamientos sobre la eficacia de la ley y sobre la asistencia psicológica y social que allí se brinda para solucionar esta problemática, puesto que, a su juicio, la solución no es de naturaleza punitiva, sino de orden preventivo.

En cuanto a la aplicación de los principios de justicia restaurativa, los funcionarios, en su mayoría, consideran que ésta se lleva a cabo a través de la conciliación, de la aplicación del principio de oportunidad y de la remisión al servicio de psicología. En este sentido, entienden la justicia restaurativa como el procedimiento dirigido a restablecer los derechos de las víctimas, a buscar una indemnización económica, a la presentación de solicitud de perdón por parte del victimario y a la iniciación de un proceso terapéutico. 
De acuerdo con los resultados, ni los funcionarios ni los usuarios del CAVIF tienen conocimientos sobre la justicia restaurativa en términos conceptuales. En su gran mayoría, los usuarios pretenden que la institución realice acciones de diverso orden para que cese la violencia y que el victimario cambie su forma de actuar. Otros, por el contrario, consideran que los usuarios buscan castigar al victimario a través de la denuncia ante las autoridades.

Para los profesionales del CAVIF, la falta de apoyo por parte del Estado para implementar programas de justicia restaurativa y brindar mayor asistencia psicológica y social para ampliar la participación de otras entidades, hace necesario un seguimiento más riguroso de este proceso con el fin de medir la reincidencia de los victimarios y verificar el cumplimiento de los acuerdos firmados. Piensan que esto último incide en la presentación continua de conciliaciones que no resuelven el conflicto, sino que, por el contrario, lo mantienen en un círculo vicioso, incidiendo en la poca credibilidad de los usuarios respecto a las instituciones.

En cuanto a la participación de la comunidad en la aplicación de justicia restaurativa, los funcionarios del CAVIF consideran que hace falta más información para la comunidad. Piensan que no se aborda esta problemática desde el punto de vista macro, sino que se queda en la esfera de lo familiar. Señalan, igualmente, la necesidad de brindar información sobre los diferentes programas que la Alcaldía lleva a cabo, con el fin de propiciar mayor participación ciudadana en la denuncia de estos actos violentos en la comunidad.

La mayoría de funcionarios no sabe en qué consiste un programa de justicia restaurativa; algunos de ellos consideran que se trata de la realización de las conciliaciones y las consecuencias legales de los acuerdos que allí se presentan, tales como: indemnización, perdón y compromiso con la víctima de no repetir la conducta. En su mayoría, opinan que a la víctima se le puede habilitar y dar protagonismo a través de acciones como: la asistencia psicológica, la información adecuada y un trato amable y coherente con la situación que se denuncia. Piensan que los usuarios deben par- ticipar de manera activa en la solución de su conflicto, proporcionando a los Fiscales las posibles soluciones que se puedan implementar.

Es importante señalar la necesidad de llevar a cabo actividades de información con la comunidad que permitan la participación activa de los colegios e instituciones sociales en general, con el fin de hacer más visible esta problemática, lograr una sensibilización sobre el problema de la violencia intrafamiliar e involucrar activamente a la comunidad en la resolución de los conflictos.

Para los profesionales del CAVIF, el personal que trabaje con un enfoque enmarcado en la justicia restaurativa debe tener una capacidad de comprensión y compromiso con los usuarios y con la problemática que allí se atiende. Consideran necesaria una capacitación especial para atender este tipo de conflictos desde una perspectiva humana, ya que por su condición de abogados, según señalan, no han sido preparados para ello. En su opinión, hay una necesidad de recibir capacitación en áreas como: psicología, asistencia social, negociación y resolución de conflictos.

En general, los funcionarios señalan que la naturaleza de la violencia intrafamiliar hace imprescindible la participación de psicólogos, trabajadores sociales, representantes de la Personería y la Procuraduría, de la Policía, de la Defensoría Pública, de Bienestar Familiar y de las Comisarías de Familia, así como la conformación de equipos interdisciplinarios que posibiliten la acción conjunta para restablecer los derechos y reparar a las víctimas. En suma, la realización de un trabajo intersectorial, interinstitucional e interdisciplinario.

Para la realización de su tarea, los Fiscales señalan que hacen falta recursos y herramientas tales como: instalaciones adecuadas, instrumentos tecnológicos, personal capacitado, espacio físico para la realización de talleres y para la ejecución de entrevistas donde se permita mantener la privacidad. Asimismo, señalan la falta de compromiso de las Entidades Promotoras de Salud (EPS) y del apoyo de las instituciones relacionadas con la problemática de familia, ya que sólo se cuenta con un defensor de familia. 
Las entrevistas aplicadas a los usuarios evidencian un desconocimiento frente a las funciones y competencias de la Fiscalía General de la Nación y el CAVIF; en algunos casos refrieren información incompleta suministrada por terceros no cualificados. En general, los usuarios poseen un conocimiento equivocado y no fundamentado frente a la institución. Los veinte usuarios entrevistados asistían al CAVIF por primera vez, aun cuando ya tenían una historia de maltrato.

Las entrevistas aplicadas a los usuarios narran situaciones de violencia caracterizadas por desigualdad de poder en la relación, continuidad en el tiempo y la sensación de amenaza a su integridad. Los usuarios describen ciclos de violencia marcados por intentos de solución fallida del conflicto, compromisos de cambio, expresiones de arrepentimiento e intervención de terceros como familiares, instituciones y asesoría profesional. Además, se evidencia una desesperanza ante la solución del conflicto por medio de institucionales tradicionales como las Comisarías de Familia y de acciones como la conciliación.

Otras respuestas se asocian con la búsqueda de apoyo y protección, y la cesación de los episodios de maltrato. En su mayoría, los usuarios mencionan que quedarían satisfechos frente al conflicto si hubiese restricciones para el victimario (para acercarse a la víctima o a los demás miembros de la familia), cumplimiento de las obligaciones económicas y modificación del comportamiento del agresor. En relación con la pena de prisión como posible castigo para el delito de violencia intrafamiliar, los usuarios expresan acuerdo en su mayoría, sólo cuando se han implementado otros mecanismos de solución y ninguno ha sido eficaz. Finalmente, asocian la pena de prisión con sentimientos de resentimiento con el agresor y pocas probabilidades de cambio.

Las entrevistas evidencian un desconocimiento de los usuarios frente a los procedimientos generales de los programas y mecanismos de justicia restaurativa. Asocian la reparación con términos como: arreglar, remediar, cambiar, reponer y, en menor frecuencia, pagar, beneficios y reconstruir. Asimismo, asocian la percepción del daño con: humillante, grave, psicológico, irreparable, miedo, dolor. Mencionan como relevante, dentro del proceso, el conocimiento de la verdad de los hechos y como sintomatología a nivel psicológico: ansiedad, depresión y baja autoestima.

En cuanto a la responsabilidad en el conflicto, la respuesta más frecuente es la admisión de responsabilidad de víctima y victimario en la presentación del conflicto. Los usuarios relacionan la admisión de responsabilidad con: permitir, tolerar y no hacer nada ante los hechos de violencia.

La mayoría de ellos considera que la persona con quien tienen el conflicto se siente culpable o responsable. Las narraciones se enmarcan en la fase de arrepentimiento del ciclo de violencia, en la que el agresor trata de recuperar el equilibrio de la relación por medio de justificaciones, manifestaciones de cariño y promesas de no recaer, con el objetivo de conciliar y continuar con su pareja.

Los usuarios, casi en su totalidad, estarían dispuestos a reunirse con el agresor en un contexto seguro y estructurado, con el objetivo de que suscitar el reconocimiento de su responsabilidad y la terminación del conflicto. Sin embrago, manifiestan que no estarían dispuestos a recibir o solicitar disculpas, asociando estos eventos con soluciones fallidas al conflicto.

En su mayoría, consideran que la comunidad podría aportar sólo en el caso de instituciones o personas que tuvieran la función de mediadores; asocian la participación de la comunidad con pérdida de privacidad. Manifiestan que están dispuestos a asistir al CAVIF de una manera periódica con la expectativa de una solución efectiva al conflicto centrada en el aspecto legal. En las respuestas se evidencia que algunas de las dificultades para la asistencia son los horarios y los deberes laborales.

Una vez identificadas las percepciones de los funcionarios del CAVIF sobre la justicia restaurativa, sus implicaciones y procedimientos, y tenidas en cuenta las representaciones de usuarios y funcionarios sobre la violencia intrafamiliar, entre otros temas conexos como la institucionalidad, las me- 
didas punitivas y algunos de orden más subjetivo como la vivencia de la violencia por parte de las víctimas del delito, se proponen algunos puntos de análisis para pensar un programa de Justicia Restaurativa en el CAVIF, de acuerdo con los fundamentos y principios que la sustentan. Aunque la propuesta se circunscribe en un escenario particular y se plantea frente a una problemática específica, se espera que sus resultados sirvan de insumo para programas de justicia restaurativa en otros escenarios de administración de justicia.

\section{Descripción de criterios}

Dada la novedad de la Justicia Restaurativa en Colombia y su reciente ingreso en el sistema de investigación judicial, es importante implementar programas con la participación de las poblaciones objetivo. Asimismo, se deben identificar aspectos fundamentales como: criterios de participación en el programa, mecanismos de participación de la comunidad, posibles acuerdos de reparación y estrategias de seguimiento y apoyo comunitario, entre otros inherentes a la implementación del mecanismo.

Capacitación con funcionarios: debido al desconocimiento casi generalizado de los funcionarios sobre la justicia restaurativa, sus implicaciones, procedimientos y principios conceptuales, es fundamental que un proyecto piloto abarque una primera fase de capacitación con los profesionales del CAVIF que estarían directamente a cargo de su implementación y conducción, y con los funcionarios de las instituciones conexas (actualmente presentes en el (AVIF) que apoyarían su desarrollo desde otros frentes (acuerdos de reparación, restablecimiento de derechos, apoyo social a las víctimas).

Información a las víctimas: debido al poco conocimiento que existe entre las víctimas de violencia intrafamiliar sobre las alternativas de abordaje jurídico-penal del delito, sus derechos y efectos jurídicos de los acuerdos, es importante elaborar dispositivos de información y estrategias de comunicación para dar a conocer los objetivos de los programas de Justicia Restaurativa que puede ofrecer el CAVIF: sus efectos, implicaciones, procedimientos y fundamentos. También es importante desarrollar propuestas acerca de cómo la conciliación podría adoptar los principios de la justicia restaurativa para lograr acuerdos efectivos y procesos adecuados de reparación. Esta información debe hacer énfasis en el carácter reparador (no punitivo) del mecanismo, en la participación voluntaria de los implicados en el delito y en la participación de la comunidad.

Información a los agresores de VIF: esta estrategia de información también debe estar dirigida a los agresores de violencia intrafamiliar, haciendo hincapié en sus derechos, condiciones de participación voluntaria, posibles estrategias de resarcimiento o acuerdos de reparación, participación de la comunidad y objetivos principales de la Justicia Restaurativa.

La comunidad: teniendo en cuenta la importancia de la participación de la comunidad en los procesos de justicia restaurativa y la idea que aún prevalece de la violencia intrafamiliar como un asunto privado, es importante adelantar procesos de información a la comunidad acerca de la función simbólica, reparadora y de control social informal que desempeña en los procedimientos de justicia. La capacitación de facilitadores comunitarios (maestros, líderes, promotores de salud, jueces de paz) capaces de orientar a víctimas y agresores y coordinar la participación de la comunidad, hace parte de esta estrategia.

Orientación psicojurídica: generar espacios informativos para víctimas y agresores de violencia intrafamiliar que deseen hacer parte del programa. En este espacio se debe suministrar toda la información relacionada con el mecanismo, sus principios, efectos y las consecuencias jurídicas derivadas del acuerdo y el proceso. La orientación que se brinde en este espacio debe conducir a las partes a tomar una decisión autónoma e informada sobre su participación en el programa.

Tipología: teniendo en cuenta lo establecido en el sistema de justicia actual, se propone desarrollar una práctica que contemple la adopción de los principios de Justicia Restaurativa en el mecanismo de la conciliación. 
Componentes: debe haber claridad en cuanto a objetivos, viabilidad, condiciones de acceso, estrategias de participación de la comunidad, acuerdos de reparación, mecanismos de protección de derechos, estrategias de seguimiento, tiempos de duración del proceso y metodología. El seguimiento que se efectúe debe permitir evaluar periódicamente el proceso y la efectividad del mismo.

Momento procesal: se debe tener en cuenta la aplicación del principio de oportunidad y la naturaleza del principio de reparación integral.

Acuerdos de reparación o estrategias de resarcimiento: se deben definir claramente los diferentes tipos de acuerdos de reparación que contempla la justicia restaurativa, teniendo en cuenta el marco normativo del sistema de justicia actual. Estos acuerdos deben contemplar la reparación económica a la víctima, pero bajo ninguna circunstancia deben reducirse a ella.

Los participantes: se deben establecer las competencias y funciones de los profesionales y las responsabilidades de las instituciones que hagan parte del proceso de acuerdo con el mecanismo de la conciliación.

En la Justicia Restaurativa es importante avanzar con respecto a la creación de programas de atención a víctimas que contemplen la dimensión psicológica, social y jurídica de los efectos del delito. Estos programas deben hacer parte de redes de apoyo y asistencia psicosocial para víctimas de violencia intrafamiliar, las cuales deben estar integradas por instituciones públicas y privadas.

Entre otros programas, se deben contemplar acciones de apoyo a niños, niñas, adolescentes y adultos mayores víctimas de violencia intrafamiliar y programas de apoyo a personas adictas al alcohol o a Sustancias Psicoactivas (SPA). Los servicios comunitarios deben integrar en esta red a los agresores de violencia intrafamiliar. Si se consideran los efectos jurídicos del proceso restaurativo (acuerdos) y la posible continuidad de la convivencia familiar e inserción social del agresor en la comunidad, es fundamental crear progra- mas de apoyo y orientación para los agresores en el seno de la misma red institucional que haya elaborado los programas de asistencia a víctimas. Los programas de asistencia a los familiares de los agresores son fundamentales, tanto por el apoyo psicosocial, como por la prevención del delito. También se debe tener en cuenta a la comunidad, tanto en el proceso de reparación, como en el ejercicio del control social informal.

Al considerar la notable disposición institucional para el desarrollo de programas de justicia restaurativa y la red institucional que actualmente opera en el CAVIF, es importante avanzar en la formulación de programas de apoyo adicionales, tales como: paneles de ayuda para jóvenes, comunidades terapéuticas, círculos de apoyo a víctimas, servicios comunitarios relacionados, programas de capacitación e inserción laboral para víctimas y programas de sensibilización comunitaria, entre otros. El programa que se implemente en el CAVIF debe estar sujeto a evaluaciones periódicas, sobre la base de su impacto en la problemática, los usuarios y la comunidad.

\section{Conclusiones}

La construcción de un programa de Justicia Restaurativa para atender casos de violencia intrafamiliar en el CAVIF debe hacer visible la misión, los objetivos y estrategias de la Fiscalía General de la Nación, así como las diferentes alternativas para abordar los conflictos intrafamiliares ante los usuarios. El poco conocimiento que existe entre las víctimas de VIF sobre las alternativas de abordaje jurídico-penal del delito, sus derechos y efectos jurídicos de los acuerdos, hace importante elaborar dispositivos de información y estrategias de comunicación para informar a los usuarios sobre los efectos, implicaciones, procedimientos y fundamentos del programa.

La capacitación a los funcionarios debe incluir la provisión de los recursos personales, técnicos y físicos para hacer viable un programa de Justicia Restaurativa en el CAVIF-, además, es importante establecer los posibles acuerdos de reparación e identificar los criterios y mecanismos de partici- 
pación de la comunidad en los procedimientos de justicia, así como las estrategias de seguimiento y apoyo comunitario.

Se deben generar espacios informativos de orientación psicojurídica para víctimas y agresores de VIF que deseen hacer parte del programa. En este espacio, se debe suministrar toda la información relacionada con el mecanismo: su definición, principios rectores, aspectos éticos, objetivos, procedimientos, efectos y consecuencias jurídicas derivadas del acuerdo. La orientación que se brinde en este espacio debe conducir a las partes a tomar una decisión autónoma e informada sobre su participación en el programa.

El programa debe apuntar a un abordaje integral de la dimensión psicológica, social y jurídica del delito. Por esta razón, debe hacer parte de redes de apoyo y asistencia psicosocial para víctimas de VIF, las cuales deben estar integradas por instituciones públicas y privadas. Los programas conexos deben estar dirigidos a niños, niñas, adolescentes y adultos mayores víctimas de VIF y personas adictas al alcohol o a SPA. Teniendo en cuenta los efectos jurídicos del proceso restaurativo y la posible continuidad de la convivencia familiar e inserción social del agresor en la comunidad, es fundamental crear programas de apoyo y orientación para los agresores en el seno de la misma red institucional de los programas de asistencia a víctimas. Los programas de asistencia a los familiares de los agresores son fundamentales, tanto por el apoyo psicosocial, como por la prevención del delito.

\section{Referencias}

Bandura, A. (1973). Aggression: A social learning analysis, Nueva Jersey: Prentice Hall. Englewood Cliffs.

Bandura, A. (1986). Social foundations of thought and action: A social-cognitive theory, Nueva Jersey: Prentice Hall, Englewood Cliffs.

Ballanga, Cánavas, Cantiè, Casanly, Alfaro, Gimeno, Guillamat, Juliá, Mercadal, Ortì, Sala \& Villabona. (2008). Implications of Personali- ty Profiles for Batterer Treatment. Journal of Interpersonal Violence, 15(5). Extraído en mayo de 2000 en http://search.ebscohost. com/login.aspx?direct=true\&db=a9h\&AN=301 5984\&site=ehost-live.

Beceiro, M. (2000). Aprendizaje de la violencia y pautas culturales: reacciones emocionales ante la violencia mediática aceptada culturalmente. Tesis de Licenciatura. A Coruña: Universidad de A Coruña.

Beristain, A. (1998). Criminología y Victimología. Alternativas Re-creadoras al Delito. Bogotá, Colombia: Leyer.

Chambersb, Ecclestona, Dayb, Wardc \& Howellsb. (2008). Treatment readiness in violent offenders: The influence of cognitive factors on engagement in violence programs. Aggression and Violent Behavior. Volume 13, Issue 4. Extraído en agosto y septiembre de 2008 en www.Sciencedirect.com.

Clemente, M. \& Espinoza, P. (2001). La mente criminal. Teorías explicativas del delito desde la psicología jurídica. Madrid, España: E. DYKINSON.

Confraternidad Carcelaria Internacional. (2005). ¿Qué es la Justicia Restaurativa? Trabajo resumen sobre Justicia Restaurativa. Extraído en agosto de 2008, de http://www.pfi.org/ cjr/spanish/quees/

Echeburúa, E. (2004). Hombres violentos contra la pareja: perfil psicopatológico y programa de intervención. Extraído en noviembre de 2008 en http://www.cej.justicia.es/pdf/publicaciones/medicos_forenses/MEDI31.pdf.

Echeburúa, Paz De Corral, Fernández \& Amor. (2004). ¿Se puede y debe tratar psicológicamente a los hombres violentos contra la pareja? Papeles del Psicólogo 57. http://www.papelesdelpsicologo.es/vernumero.asp?id=1159

Ehrensaft, Cohen, Brown, Smailes, Chen \& Johnson. (2007). Intergenerational Transmission 
of Partner Violence. A 20-Year Prospective Study. Journal of Consulting and Clinical Psychology 71 (4).

Eron, L.,Walder y Lefkowitz. (1971). Learning aggression in Children. Boston: Little Brow.

Fariña, Arce \& Novo. (2008). Estado clínico y tratamiento penitenciario en penados en tercer grado por violencia de género. En Rodríguez, Bringas, Fariña, Arce, \& Bernardo (Eds.), Psicología Jurídica. Entorno judicial y delincuencial, 5. Colección Psicología y Ley. Tomo 5. Sociedad Española de Psicología Jurídica y Forense. Ediciones de la Universidad de Oviedo, O. C.

Ferrer, V., Bosch, E., García, E., Manassero, A. \& Gili, M. (2004). Estudio meta-analítico de características diferenciales entre maltratadores y no maltratadores: El caso de la psicopatología y el consumo de alcohol o drogas. PSYKHE, 13 (1), 141-156. Extraído en junio de 2008 en http://search.ebscohost.com/login. aspx?direct=true \&db=psyh\&AN=2005-00049012\&amp;lang=es\&site=ehost-live.

Fiscalía General de la Nación. (2004). Acuerdo 123 de 2004. Extraído en julio de 2008 en http:// www.fiscalia.gov.co/pag/entidad/quehacer. html.

Fiscalía General de la Nación. (2004). Ley 906 de 2004. Extraído en julio de 2008 en http://www. fiscalia.gov.co/pag/entidad/quehacer.html.

Fiscalía General de la Nación. (2005). Manual de procedimientos de Fiscalía en el Sistema Penal Acusatorio Colombiano. Extraído en julio de 2008 en http://www.justiciarestaurativa. com/Documentos/ManualProcedimientoFiscalia.pdf.

Garrido, Stangeland y Redondo. (2006). Principios de criminología. ( $3^{\text {a }}$. ed.) Valencia: Tirand lo Blanch.

García-Pablos, A. (1999). Tratado de criminología. Valencia: Tirant lo Blanch.
Gray.J.A. (1992). La Psicología del miedo y del estrés. Barcelona: Paidós

Jennings, J. \& Murphy, M. (2000). Psychology of Men and Male-Male Dimensions of MaleFemale Battering: A New Look at Domestic Violence. Psychology of Men and Masculinity: The Educational Publishing Foundation, 1 (1), 21-29.

Labrador, F., De Luis P. Paz, R. \& Fernández, R. (2008). Mujeres víctimas de la violencia doméstica. Madrid: Ediciones Pirámide.

Lila M., Herrero, J. \& Gracia, E. (2008). Atribución de responsabilidad y minimización en hombres penados por violencia contra la mujer: un instrumento de evaluación en Rodríguez, Bringas, Fariña, Arce \& Bernardo (Eds.). Psicología Jurídica. Familia y Victimología, 6. Colección Psicología y Ley. Tomo 6. Sociedad Española de Psicología Jurídica y Forense. Ediciones de la Universidad de Oviedo. O. C.

Lorenz, K. (1976). Sobre la agresión, el pretendido mal. Madrid: Siglo XXI.

Madina, J. (2005). Perfil psicológico e intervención de los hombres maltratadores. Gizonezkoak genero indarkeriaren aurrean. En: Los hombres ante la violencia de género. Departamento para los Derechos Humanos, el Empleo y la Inserción Social, Guatemala. Extraído en noviembre de 2008 en www.Sciencedirect.com.

Medina, Aracena, M. \& Bilboa, M. (2004). Maltrato físico infantil y apoyo social: Un estudio descriptivo-comparativo entre maltratadores y no maltratadores de la ciudad de Temuco. Chile.

Neuman, J.P., Kosson, D.S. \& Paatterson. (1992). Delay of gratification in Psychopathic and nonpsychopathic offenders. Journal of $a b$ normal Psychology, 101(4), 630-636.

Ortiz, M. A. (2005). Violencia doméstica: modelo multidimensional y programa de intervención. Extraído en septiembre de 
2008 en Universidad Complutense de Madrid. http://site.ebrary.com/lib/unalbogsp/ Doc?id=10234553\&ppg=465

Pueyo, A. \& Redondo, S. (2007). Predicción de la violencia: Entre la peligrosidad y la valoración del riesgo de violencia. Papeles del psicólogo, 28 (3), 157-173.

Ribero, R. \& Sánchez, F. (2004). Determinantes, efectos y costos de la violencia intrafamiliar en Colombia, Documento de Derechos Humanos de las Mujeres 2004: Los derechos civiles y políticos de las mujeres colombianas a la luz de la observación general. Extraído en octubre de 2008 en Atarraya.Org/Article. Php3?Id_Article=143.

Rodríguez, Azcárate, Echauri \& Martínez. (2008). Los programas de terapia individual y de grupo con agresores en el ámbito familiar. En Rodríguez, Bringas, Fariña, Arce \& Bernardo (Eds.). Psicología Jurídica. Familia y Victimología, 6. Colección Psicología y Ley. Tomo 6. Sociedad Española de Psicología Jurídica y Forense. Ediciones de la Universidad de Oviedo. O. C.

Ruiz, A. \& Expósito, J. (2008). Intervención especifica con internos condenados por delito de violencia de género. En Rodríguez, Bringas, Fariña, Arce \& Bernardo (Eds.) Psicología Jurídica. Familia y Victimología, 6. Tomo 6. Colección Psicología y Ley. Sociedad Española de Psicología Jurídica y Forense. Ediciones de la Universidad de Oviedo. O. C.
Salazar, Emshoff, Baker \& Crowley. (2007). Examining the behavior of a system: An outcome evaluation of a coordinated community response to domestic violence. Fam Viol, 22, DOI 10.1007/s10896-007-9116.

Smith, M. (2007). Self-Deception Among Men Who Are Mandated to Attend a Batterer Intervention Program is Clinical Associate. Perspectives in Psychiatric Care, 43 (4), october.

Soria, M. \& Rodríguez, C. (2003). Perfil Psicológico del homicida doméstico. Extraído en septiembre de 2008 en el Colegio Oficial Psicólogos de Madrid. http.//site.ebrary.com/lib/unalbogsp/ Doc?id=10058510\&ppg=1http.//www.cop.es/ publicaciones /jurìdica /jurìdica. Htm.

Traverso, M. T. (2007).Violencia en la pareja, la cara oculta de la relación. Banco interamericano de desarrollo: New York. Extraído en septiembre de 2008 en www.iadb.org/pub ISBN-1886938-78-4.

Walker, L.E. (1991): Pos-traumatic stress disorder in women: diagnosis and treatment of battered woman syndrome, 281(1), 21-29.

Walgrave, L. (1999). La justice restaurative: à la recherche d'une théorie et d'un programme. Criminologie, 32,(1), Belgique. 
